\title{
ACCELERATION OF CONVERGENCE OF A FAMILY OF LOGARITHMICALLY CONVERGENT SEQUENCES
}

\author{
ANDREW H. VAN TUYL
}

\begin{abstract}
The asymptotic behavior of several sequence transformations is investigated as $n \rightarrow \infty$ when applied to a certain family of logarithmically convergent sequences. The transformations considered are the iterations of the transformations $e_{1}^{(s)}\left(A_{n}\right)$ of Shanks and $W_{n}$ of Lubkin, the $\theta$-algorithm of Brezinski, the Levin $u$ - and $v$-transforms, and generalizations of the $\rho$ algorithm and the Neville table. Computational results are given for both real and complex sequences.
\end{abstract}

\section{INTRODUCTION}

An infinite sequence $A_{n}$ with limit $A$ is defined to be logarithmically convergent if

$$
\lim _{n \rightarrow \infty} \Delta A_{n+1} / \Delta A_{n} \text { and } \lim _{n \rightarrow \infty}\left(A_{n+1}-A\right) /\left(A_{n}-A\right)
$$

both exist and are equal to unity, where $\Delta A_{n}=A_{n+1}-A_{n}$. Such sequences are slowly convergent, and therefore not convenient for numerical calculation. When these two limits are equal to $s \neq 1$ or 0 , the convergence is called linear. Methods for accelerating logarithmic convergence have been investigated in [1] through [4], [9] through [15], [22], and in other references.

In the present paper, we will consider the family of sequences $A_{n}$ which are asymptotically of the form

$$
A_{n} \sim A+n^{-\alpha} \sum_{j=0}^{\infty} a_{j} n^{-j / k}
$$

as $n \rightarrow \infty$, where $k \geq 1$ is an integer, and $A, \alpha$, and the $a_{j}$ are complex. The expansion in (1.1) is assumed to be asymptotic in the sense of Poincaré, with $a_{0} \neq 0$ and $\alpha \neq-m / k$ or $-m / k-1$ for $m \geq 0$. We see that $A_{n}$ is logarithmically convergent when $\operatorname{Re} \alpha>0$, and divergent when $\operatorname{Re} \alpha<0$. Sequences with the asymptotic behavior (1.1) occur in applications $([7,8$, and $18]$, for example), and it can be shown that all slowly convergent sequences occurring as examples in the references of the present paper satisfy (1.1) with $k=1$. In [18], possible applications in which $k>1$ are given.

Received by the editor April 20, 1990 and, in revised form, December 14, 1992.

1991 Mathematics Subject Classification. Primary 40A25; Secondary 65B05.

Key words and phrases. Acceleration of convergence, logarithmic convergence, Levin $u$-transform, $\theta$-algorithm, $\rho$-algorithm, slowly convergent series, slowly convergent sequences, transformations of sequences.

Research supported in part by the Independent Research Program at the Naval Surface Warfare Center. 
The purpose of the present investigation is to find the asymptotic behavior of the following transformations as $n \rightarrow \infty$ when applied to the family of sequences (1.1): (a) the transformations $e_{1}^{(s)}\left(A_{n}\right)$ of Shanks and $W_{n}$ of Lubkin and their iterations; (b) the $\theta$-algorithm of Brezinski; (c) a generalization of the $\rho$-algorithm of Wynn; (d) the $u$-and $v$-transforms of Levin; and (e) a linear transformation which is a generalization of the Neville table. The generalization of the $\rho$-algorithm does not seem to have been given explicitly in the literature, but it can be shown to follow by applying results of [2] to sequences satisfying (1.1). The linear transformation in (e) appears to be new. When $k=1$, some overlap of the present results is found with [1], where $e_{1}^{(s)}\left(A_{n}\right)$ is applied to sequences of the form (1.1), with [14] in the case of the Levin transforms, and more recently, with [12]. The generalization of the $\rho$-algorithm in [12] agrees with the present one when $k=1$.

The $\theta$-algorithm, the $u$ - and $v$-transforms of Levin, and the iteration of $W_{n}$ accelerate linear as well as logarithmic convergence. Because of this property, the Levin $u$-transform has been used in a general-purpose computer program for acceleration of convergence [5]. The $\theta$-algorithm and the Levin transforms are among several procedures for acceleration of convergence tested in [16] and [17].

Each of the transformations (a) through (e) produces transformed sequences $B_{m, n}, m=1,2, \ldots$. The results of the present investigation show that for (a), (b), (c), and (e), $B_{m+1, n}$ converges more rapidly than $B_{m, n}$ as $n \rightarrow \infty$, $m \geq 1$, for all sequences $A_{n}$ satisfying (1.1). In the case of (d), however, this acceleration is found only for $k=1$. For $k \geq 2$, all sequences $B_{m, n}$ obtained from a given sequence by use of the $u$-and $v$-transforms are found to converge with the same rapidity.

In the present paper, acceleration of convergence is investigated only under the limiting process $\lim _{n \rightarrow \infty} B_{m, n}$ for fixed $m$. This process, and the limiting process $\lim _{m \rightarrow \infty} B_{m, n}$ for fixed $n$ are called Process I and Process II, respectively, in [14] and [15]. In [14] and [15] acceleration of convergence is investigated theoretically under both Process I and Process II when Levin's transformations are applied to logarithmically and linearly convergent sequences, including sequences of the form (1.1) with $k=1$.

The investigation of cases (a) through (e) is given in $\S \S 2$ through 6 of the present paper. Section 7 includes numerical examples, with both real and complex $\alpha$ and $A$, to illustrate the acceleration of convergence obtained by the transformations considered and to test the asymptotic results. As suggested by the referee, calculations have also been carried out for the $d^{(m)}$-transformation due to Levin and Sidi [10], using the algorithm and FORTRAN program of [6]. For $m=1$, the $d^{(m)}$-transformation reduces to the Levin $u$-transformation when $\sigma=$ SIGMA $=1 D 0$ and INCR $=1$ in the FORTRAN program of [6]. When $k \geq 2$ in (1.1) and $m=k, \sigma=1$, the present calculations indicate that acceleration of convergence occurs mainly under Process II.

\section{The tRANSFORMATIONS $e_{1}^{(s)}\left(A_{n}\right)$ AND $W\left(A_{n}\right)$}

The transformation $e_{1}^{(s)}\left(A_{n}\right)$ is defined by

$$
e_{1}^{(s)}\left(A_{n}\right)=\frac{s e_{1}\left(A_{n}\right)-A_{n}}{s-1},
$$


$s \neq 1$, where

$$
e_{1}\left(A_{n}\right)=\frac{A_{n-1} A_{n+1}-A_{n}{ }^{2}}{A_{n-1}+A_{n+1}-2 A_{n}}=A_{n}+\left[\Delta\left(1 / \Delta A_{n-1}\right)\right]^{-1}
$$

is the first-order transform of Shanks [13, p. 39], also called the Aitken $\delta^{2}$ process. The transformation (2.1) was introduced to accelerate the convergence of $A_{n}$ when the conditions

$$
\lim _{n \rightarrow \infty} \Delta A_{n+1} / \Delta A_{n}=1, \quad \lim _{n \rightarrow \infty} \Delta e_{1}\left(A_{n}\right) / \Delta A_{n}=1 / s \neq 1
$$

are satisfied simultaneously. A transformation equivalent to (2.1), called $U_{n}$, was also introduced by Lubkin [11, p. 232]. A second transformation due to Lubkin for acceleration of convergence of sequences satisfying (2.3), called $W_{n}$ $\left[11\right.$, p. 229], does not require knowledge of $s$. It will be denoted by $W\left(A_{n}\right)$ in the following, and is given by

$$
W\left(A_{n}\right)=\frac{e_{1}\left(A_{n}\right)-A_{n} \Delta e_{1}\left(A_{n}\right) / \Delta A_{n}}{1-\Delta e_{1}\left(A_{n}\right) / \Delta A_{n}} .
$$

We will use the notation $e_{1}^{\left(s_{1}\right)}\left(e_{1}^{\left(s_{2}\right)}\left(A_{n}\right)\right)=e_{1}^{\left(s_{1}\right)} e_{1}^{\left(s_{2}\right)}\left(A_{n}\right)$ and $W\left(W^{m-1}\left(A_{n}\right)\right)=$ $W^{m}\left(A_{n}\right)$, where $W^{1}\left(A_{n}\right)=W\left(A_{n}\right)$. Using the second expression in (2.2), we find that (2.1) and (2.4) can be written in the alternate forms

$$
e_{1}^{(s)}\left(A_{n}\right)=A_{n}+\frac{s}{s-1}\left[\Delta\left(1 / \Delta A_{n-1}\right)\right]^{-1}
$$

and

$$
W\left(A_{n}\right)=A_{n}-\frac{\Delta A_{n}\left[\Delta\left(1 / \Delta A_{n-1}\right)\right]^{-1}}{\Delta\left[\Delta\left(1 / \Delta A_{n-1}\right)\right]^{-1}},
$$

respectively. The proofs of the following theorems will be carried out by direct substitution, noting that asymptotic expansions can always be added and multiplied, and that division can be carried out when the leading coefficient of the divisor does not vanish.

Theorem 2.1. Let $A_{n}$ satisfy the conditions of (1.1), and let $s \neq 1$. Then as $n \rightarrow \infty$, we have

$$
\begin{aligned}
e_{1}^{(\alpha+1)}\left(A_{n}\right) & \sim A+n^{-\alpha-2} \sum_{j=0}^{\infty} b_{j} n^{-j}, \quad k=1 \\
& \sim A+n^{-\alpha-1 / k} \sum_{j=0}^{\infty} c_{j} n^{-j / k}, \quad k \geq 2
\end{aligned}
$$

and

$$
e_{1}^{(s)}\left(A_{n}\right) \sim A+n^{-\alpha} \sum_{j=0}^{\infty} d_{j} n^{-j / k}, \quad s \neq \alpha+1,
$$

where $b_{0}$ and $c_{0}$ may vanish, and $d_{0} \neq 0$. 
Proof. We have

$$
\Delta A_{n} \sim n^{-\alpha-1} \sum_{j=0}^{\infty} e_{j} n^{-j / k}
$$

for $k \geq 1$, where $e_{0}=-\alpha a_{0} \neq 0$. Considering the case $k=1$ first, we find that

$$
\left[\Delta\left(1 / \Delta A_{n-1}\right)\right]^{-1} \sim n^{-\alpha} \sum_{j=0}^{\infty} f_{j} n^{-j}
$$

where

$$
\begin{aligned}
& f_{0}=-\frac{\alpha a_{0}}{\alpha+1}, \\
& f_{1}=-\frac{\alpha a_{1}}{\alpha+1}, \\
& f_{2}=-\frac{\alpha(\alpha-1)}{12(\alpha+1)} a_{0}-\frac{(\alpha-1)(\alpha+2)}{(\alpha+1)^{2}} a_{2}-\frac{1}{\alpha(\alpha+1)} \frac{a_{1}{ }^{2}}{a_{0}} .
\end{aligned}
$$

The first part of (2.7) follows by substitution of (1.1), (2.10), and (2.11) into (2.5), with

$$
b_{0}=-\frac{\alpha-1}{12} a_{0}+\frac{2}{\alpha(\alpha+1)} a_{2}-\frac{1}{\alpha^{2}} \frac{a_{1}^{2}}{a_{0}} .
$$

We see that $b_{0}$ may vanish for a given $\alpha \neq-1$ or 0 .

When $k \geq 2$, we have

$$
\left[\Delta\left(1 / \Delta A_{n-1}\right)\right]^{-1} \sim n^{-\alpha} \sum_{j=0}^{\infty} g_{j} n^{-j / k},
$$

where

$$
\begin{aligned}
& g_{0}=-\frac{\alpha a_{0}}{\alpha+1}, \\
& g_{1}=-\frac{(\alpha+1 / k)(\alpha+1-1 / k)}{(\alpha+1)^{2}} a_{1} .
\end{aligned}
$$

The statement of (2.7) for $k \geq 2$ now follows from (1.1), (2.13), and (2.14), with

$$
c_{0}=\frac{(1-1 / k) / k}{\alpha(\alpha+1)} a_{1} .
$$

We see that $c_{0}$ vanishes when $a_{1}=0$ for $\alpha \neq-1$ or 0 .

To prove the last part of the theorem, we find from (2.10) through (2.14) that $(2.8)$ holds with

$$
d_{0}=\frac{s-\alpha-1}{(s-1)(\alpha+1)} a_{0}
$$

when $s \neq 1$ and $s \neq \alpha+1$. We see that $d_{0} \neq 0$. 
Corollary 2.1.1. For fixed $m \geq 0$ and $k \geq 1$, we have

$$
\begin{aligned}
e_{1}^{(\alpha+1+m / k)} e_{1}^{(\alpha+1+(m-1) / k)} & \cdots e_{1}^{(\alpha+1)}\left(A_{n}\right) \\
& \sim A+n^{-\alpha-(m+1) / k} \sum_{j=0}^{\infty} b_{m, j} n^{-j / k},
\end{aligned}
$$

where $b_{2 m, 0}=0$ for $m \geq 0$ when $k=1$, and where $b_{m, 0}$ may also vanish for other values of $m$ when $k \geq 1$.

Proof. It follows from Theorem 2.1 that (2.17) holds for $m=0$, since $a_{0} \neq 0$, with $b_{0,0}=0$ when $k=1$. Assuming that it holds for $0 \leq m \leq m_{1}$, let $b_{m_{1}, j}=0$ for $0 \leq j \leq p-1$ for some integer $p \geq 1$, and let $b_{m_{1}, p} \neq 0$. It then follows from Theorem 2.1 that (2.17) holds for $m_{1}+1 \leq m \leq m_{1}+p$, with the first $p-\left(m-m_{1}\right)-1$ coefficients equal to zero for each $m$ in this interval. We can now repeat this process starting from $m=m_{1}+1$, since $b_{m_{1}, 0} \neq 0$. Hence, (2.17) holds for all $m \geq 0$. When $k=1$, it then follows from (2.7) and (2.8) that $b_{2 m, 0}=0$ for $m \geq 0$.

Corollary 2.1.2. When $k=1$,

$$
\begin{aligned}
& e_{1}^{(\alpha+2 m+1)} e_{1}^{(\alpha+2 m-1)} \cdots e_{1}^{(\alpha+1)}\left(A_{n}\right) \\
& \sim A+n^{-\alpha-2 m-2} \sum_{j=0}^{\infty} b_{m, j} n^{-j}, \quad k=1,
\end{aligned}
$$

for $0 \leq m \leq m_{1}$, where $m_{1}$ is the first value of $m$ for which $b_{m_{1}+1,0}$ vanishes.

This corollary is an immediate consequence of Theorem 2.1. We see that Corollary 2.1.1 holds for all $m \geq 0$, while Corollary 2.1.2 may hold only for a finite number of values of $m$. When $k=1$ and (2.18) is applicable, however, the latter should be both more efficient and more accurate than (2.17).

Theorem 2.2. Let $A_{n}$ satisfy the conditions of (1.1). Then as $n \rightarrow \infty$,

$$
\begin{aligned}
W\left(A_{n}\right) & \sim A+n^{-\alpha-2} \sum_{j=0}^{\infty} b_{j} n^{-j}, \quad k=1 \\
& \sim A+n^{-\alpha-1 / k} \sum_{j=0}^{\infty} c_{j} n^{-j / k}, \quad k \geq 2,
\end{aligned}
$$

where $b_{0}$ and $c_{0}$ may vanish.

Proof. Considering the case $k=1$ first, we find from (2.9) through (2.12) that

$$
\frac{\Delta A_{n}\left[\Delta\left(1 / \Delta A_{n-1}\right)\right]^{-1}}{\Delta\left[\Delta\left(1 /\left(\Delta A_{n-1}\right)\right]^{-1}\right.} \sim n^{-\alpha} \sum_{j=0}^{\infty} d_{j} n^{-j},
$$

where

$$
\begin{aligned}
& d_{0}=a_{0}, \\
& d_{1}=a_{1}, \\
& d_{2}=\frac{1-\alpha}{6 \alpha} a_{0}+\frac{(\alpha+2)\left(\alpha^{2}-\alpha+2\right)}{\alpha^{2}(\alpha+1)} a_{2}-\frac{2}{\alpha^{3}} \frac{a_{1}^{2}}{a_{0}} .
\end{aligned}
$$


We see that the first part of (2.19) follows from (1.1), (2.6), (2.20), and (2.21), with

$$
b_{0}=\frac{\alpha-1}{6 \alpha} a_{0}-\frac{4}{\alpha^{2}(\alpha+1)} a_{2}+\frac{2}{\alpha^{3}} \frac{a_{1}^{2}}{a_{0}} .
$$

We see that the ratio of $(2.22)$ to $(2.12)$ is equal to $2 / \alpha$. Hence, if $b_{0}$ vanishes in (2.12), it also vanishes in (2.22). When $k \geq 2$, we have

$$
\frac{\Delta A_{n}\left[\Delta\left(1 / \Delta A_{n-1}\right)\right]^{-1}}{\Delta\left[\Delta\left(1 / \Delta A_{n-1}\right]^{-1}\right.} \sim n^{-\alpha} \sum_{j=0}^{\infty} e_{j} n^{-j / k},
$$

where

$$
\begin{aligned}
& e_{0}=a_{0}, \\
& e_{1}=\frac{(\alpha+1 / k)\left[\alpha^{2}+(1-1 / k) \alpha-(1-1 / k) / k\right]}{\alpha^{2}(\alpha+1)} a_{1} .
\end{aligned}
$$

The second part of (2.19) follows from (1.1), (2.6), (2.23), and (2.24), with

$$
c_{0}=\frac{(1-1 / k) / k^{2}}{\alpha^{2}(\alpha+1)} a_{1} \text {. }
$$

We see that $c_{0}$ vanishes when $a_{1}=0$ for $\alpha \neq-1$ or 0 .

Corollary 2.2. As $n \rightarrow \infty$ for fixed $m \geq 1$, we have

$$
\begin{aligned}
W^{m}\left(A_{n}\right) & \sim A+n^{-\alpha-2 m} \sum_{j=0}^{\infty} b_{m, j} n^{-j}, \quad k=1 \\
& \sim A+n^{-\alpha-m / k} \sum_{j=0}^{\infty} c_{m, j} n^{-j / k}, \quad k \geq 2,
\end{aligned}
$$

where $b_{m, 0}$ and $c_{m, 0}$ may vanish for some values of $m$.

Proof. When $k=1$, it follows from Theorem 2.2 that (2.26) holds through the first value $m_{1}$ of $m$ for which $b_{m_{1}, 0}=0$. Let $b_{m_{1}, j}=0$ for $0 \leq j \leq p-1$, with $b_{m_{1}, p} \neq 0$. Then we have

$$
W^{m_{1}}\left(A_{n}\right) \sim A+n^{-\alpha-p-2 m_{1}} \sum_{j=0}^{\infty} b_{m_{1}, j}^{\prime} n^{-j},
$$

where $b_{m_{1}, j}^{\prime}=b_{m_{1}, j+p}$, and $b_{m_{1}, 0}^{\prime} \neq 0$. We can therefore apply Theorem 2.2 again with $\alpha$ replaced by $\alpha+p$. Continuing in this way, we obtain the first part of (2.26) with some of the coefficients equal to zero. Similarly, when $k \geq 2$, let $c_{m_{1}, j}=0$ for $0 \leq j \leq p-1$, with $c_{m_{1}, 0} \neq 0$. Then we have

$$
W^{m_{1}}\left(A_{n}\right) \sim A+n^{-\alpha-p / k-m_{1} / k} \sum_{j=0}^{\infty} c^{\prime}{ }_{m_{1}, j} n^{-j / k},
$$

where $c^{\prime} m_{1}, j=c_{m_{1}, j+p}$, and $c_{m_{1}, 0}^{\prime} \neq 0$. As before, we obtain the second part of (2.26) with some of the coefficients equal to zero. 
3. THE $\theta$-ALGORITHM

The $\theta$-algorithm of Brezinski is defined by the equations

$$
\theta_{-1}^{(n)}=0, \quad \theta_{0}^{(n)}=A_{n}
$$

and

$$
\begin{gathered}
\theta_{2 m+1}^{(n)}=\theta_{2 m-1}^{(n)}+1 / \Delta \theta_{2 m}^{(n)}, \\
\theta_{2 m+2}^{(n)}=\theta_{2 m}^{(n+1)}-\frac{\Delta \theta_{2 m}^{(n+1)}\left[\Delta\left(1 / \Delta \theta_{2 m+1}^{(n)}\right)\right]^{-1}}{\Delta\left[\Delta\left(1 / \Delta \theta_{2 m+1}^{(n)}\right)\right]^{-1}}
\end{gathered}
$$

for $m \geq 0[4$, p. 34], where $\Delta$ is the forward difference operator acting on $n$. The following theorem will now be proved by use of Theorem 2.2.

Theorem 3.1. Let $A_{n}$ satisfy the conditions of (1.1). Then as $n \rightarrow \infty$ for fixed $m \geq 1$, we have

$$
\begin{aligned}
\theta_{2 m}^{(n)} & \sim A+n^{-\alpha-2 m} \sum_{j=0}^{\infty} b_{m, j} n^{-j}, \quad k=1 \\
& \sim A+n^{-\alpha-m / k} \sum_{j=0}^{\infty} c_{m, j} n^{-j / k}, \quad k \geq 2
\end{aligned}
$$

and

$$
\begin{aligned}
\theta_{2 m-1}^{(n)} & \sim n^{\alpha+2 m-1} \sum_{j=0}^{\infty} d_{m, j} n^{-j}, \quad k=1 \\
& \sim n^{\alpha+(m-1) / k+1} \sum_{j=0}^{\infty} e_{m, j} n^{-j / k}, \quad k \geq 2,
\end{aligned}
$$

where $b_{m, 0}, c_{m, 0}, d_{m, 0}$, and $e_{m, 0}$ may vanish for some values of $m$.

Proof. From (3.1) through (3.3) together with (2.9), we have

$$
\theta_{1}^{(n)}=1 / \Delta A_{n}
$$

and

$$
\theta_{2}^{(n)}=A_{n+1}-\frac{\Delta A_{n+1}\left[\Delta\left(1 / \Delta A_{n}\right)\right]^{-1}}{\Delta\left[\Delta\left(1 / \Delta A_{n}\right)\right]^{-1}}=W\left(A_{n+1}\right) .
$$

It follows from Theorem 2.2 and from (3.6) and (2.9) that (3.4) and (3.5) hold for $m=1$.

Assuming that (3.4) and (3.5) hold for a given $m$ with $b_{m, 0}$ and $c_{m, 0} \neq 0$, we find from (3.2) and (3.4) that (3.5) holds for $m+1$ with $d_{m+1,0}=1 / b_{m, 0}$, $e_{m, 0}=1 / c_{m, 0}$. In order to show that (3.4) holds for $m+1$, we find from (3.2) and (3.3) that

$$
\theta_{2 m+2}^{(n)}=\theta_{2 m}^{(n+1)}-\frac{\Delta \theta_{2 m}^{(n+1)}\left[\Delta \theta_{2 m-1}^{(n+1)}+\Delta\left(1 / \Delta \theta_{2 m}^{(n)}\right)\right]^{-1}}{\Delta\left[\Delta \theta_{2 m-1}^{(n)}+\Delta\left(1 / \Delta \theta_{2 m}^{(n)}\right)\right]^{-1}}
$$


Referring to (3.4) and (3.5), we have

$$
\begin{aligned}
& {\left[\Delta \theta_{2 m-1}^{(n+1)}+\Delta\left(1 / \Delta \theta_{2 m}^{(n)}\right)\right]^{-1}} \\
& \quad=\left[\Delta\left(1 / \Delta \theta_{2 m}^{(n)}\right)\right]^{-1}\left\{1+\Delta \theta_{2 m-1}^{(n+1)}\left[\Delta\left(1 / \Delta \theta_{2 m}^{(n)}\right)\right]^{-1}\right\}^{-1} \\
& \quad \sim\left[\Delta\left(1 / \Delta \theta_{2 m}^{(n)}\right)\right]^{-1}\left[1+n^{-2} \sum_{j=0}^{\infty} \bar{c}_{m, j} n^{-j}\right], \quad k=1 \\
& \quad \sim\left[\Delta\left(1 / \Delta \theta_{2 m}^{(n)}\right)\right]^{-1}\left[1+n^{-1 / k} \sum_{j=0}^{\infty} \bar{d}_{m, j} n^{-j / k}\right], \quad k \geq 2,
\end{aligned}
$$

where

$$
\begin{aligned}
& \bar{c}_{m, 0}=\frac{(\alpha+2 m)(\alpha+2 m-1)}{\alpha+2 m+1} b_{m, 0} d_{m, 0}, \\
& \bar{d}_{m, 0}=\frac{(\alpha+m / k)[\alpha+(m-1) / k+1]}{\alpha+m / k+1} c_{m, 0} e_{m, 0} .
\end{aligned}
$$

Substituting (3.9) into (3.8) and referring to (2.7), we obtain

$$
\begin{aligned}
\theta_{2 m+2}^{(n)} & \sim W\left(\theta_{2 m}^{(n+1)}\right)+n^{-\alpha-2 m-2} \sum_{j=0}^{\infty} \bar{e}_{m, j} n^{-j}, \quad k=1 \\
& \sim W\left(\theta_{2 m}^{(n+1)}\right)+n^{-\alpha-(m+1) / k} \sum_{j=0}^{\infty} \bar{f}_{m, j} n^{-j / k}, \quad k \geq 2,
\end{aligned}
$$

where

$$
\begin{aligned}
& \bar{e}_{m, 0}=-2\left[\frac{\alpha+2 m-1}{\alpha+2 m+1}\right] b_{m, 0}{ }^{2} d_{m, 0} \\
& \bar{f}_{m, 0}=-2\left[\frac{\alpha+(m-1) / k+1}{\alpha+m / k+1}\right] c_{m, 0} e_{m, 0} .
\end{aligned}
$$

Finally, it follows from Theorem 2.2 and from (3.9) through (3.12) that (3.4) holds for $m+1$. Since (3.4) and (3.5) hold for $m=1$, it follows that they hold up to the first value of $m$ for which $b_{m, 0}$ and $c_{m, 0}$ vanish. We note that the conditions $b_{m, 0} \neq 0$ and $c_{m, 0} \neq 0$ are necessary only for the application of Theorem 2.2, and that $d_{m, 0}$ and $e_{m, 0}$ may vanish.

To complete the proof, we first consider the case $k=1$. Assuming that (3.4) and (3.5) hold for $1 \leq m \leq m_{1}$, let $b_{m_{1}, j}=0$ for $0 \leq j \leq p-1$ for some integer $p \geq 1$, and let $b_{m_{1}, p} \neq 0$. Then writing $\alpha_{1}=\alpha+p$ when $m>m_{1}$, we have

$$
\theta_{2 m}^{(n)} \sim A+n^{-\alpha_{1}-2 m} \sum_{j=0}^{\infty} b_{m, j}^{\prime} n^{-j}
$$

and

$$
\theta_{2 m-1}^{(n)} \sim n^{\alpha_{1}+2 m-1} \sum_{j=p}^{\infty} d_{m, j}^{\prime} n^{-j}
$$

where $b_{m, j}^{\prime}=b_{m, j+p}$ and $d_{m, j}^{\prime}=d_{m, j-p}$. Since $b_{m, 0}^{\prime} \neq 0$, and since it is permissible to have $d_{m, 0}^{\prime}$ in the first part of the proof as noted above, it follows 
that a continuation of the calculation of $\theta_{m}^{(n)}$ leads to results of the form (3.4) and (3.5) with $\alpha$ replaced by $\alpha_{1}$. This asymptotic behavior then holds until $b_{m, 0}^{\prime}$ vanishes. Repeating the preceding discussion as often as necessary, we obtain results which can be written in the form (3.4) and (3.5) with certain of the coefficients equal to 0 .

Similarly, when $k \geq 2$, let $d_{m, j}=0$ for $0 \leq j \leq p-1$ with $d_{m, p} \neq 0$. Then

$$
\theta_{2 m}^{(n)} \sim A+n^{-\alpha_{1}-m / k} \sum_{j=0}^{\infty} c_{m, j}^{\prime} n^{-j / k}
$$

and

$$
\theta_{2 m-1}^{(n)} \sim n^{\alpha_{1}+(m-1) / k+1} \sum_{j=p}^{\infty} e_{m, j}^{\prime} n^{-j / k},
$$

for $m>m_{1}$, with $c_{m, j}^{\prime}=c_{m, j+p}, e_{m, j}^{\prime}=e_{m, j-p}$, and $\alpha_{1}=\alpha+p / k$. The remainder of the proof is now the same as that for the case $k=1$.

\section{A generalization of The $\rho$-Algorithm}

Wynn's $\rho$-algorithm [22] is defined by

$$
\begin{aligned}
& \rho_{-1}^{n}=0, \quad \rho_{0}^{n}=A_{n}, \\
& \rho_{s}^{n}=\rho_{s-2}^{n+1}+\frac{s}{\Delta \rho_{s-1}^{n}},
\end{aligned}
$$

$s \geq 1$, where as before, $\Delta$ is the forward difference operator acting on $n$. An analysis similar to that in $\S 3$ shows that a theorem for $\rho_{s}^{n}$ of the same form as Theorem 3.1 holds only when $\alpha=k=1$. In order to obtain acceleration of convergence of $A_{n}$ for general $\alpha$ and for $k \geq 1$, we introduce a generalization of (4.1) given by

$$
\begin{gathered}
\rho_{-1, n}^{(\alpha, k)}=0, \quad \rho_{0, n}^{(\alpha, k)}=A_{n}, \\
\rho_{s, n}^{(\alpha, k)}=\rho_{s-2, n+1}^{(\alpha, k)}+C_{s}^{(\alpha, k)} / \Delta \rho_{s-1, n}^{(\alpha, k)},
\end{gathered}
$$

$s \geq 1$, where

$$
C_{s}^{(\alpha, 1)}=\alpha+s-1
$$

and

$$
C_{2 m-1}^{(\alpha, k)}=\alpha+(m-1) / k, \quad C_{2 m}^{(\alpha, k)}=\alpha+(m-1) / k+1
$$

for $m \geq 1, k \geq 2$. We see that the algorithm (4.2) through (4.4) reduces to the $\rho$-algorithm when $\alpha=k=1$, and hence, that $\rho_{s, n}^{(1,1)}=\rho_{s}^{n}$. This algorithm can be arrived at by inspection, starting from (2.5) and Theorem 2.1. Alternatively, it can be obtained by the procedure of [2, p. 158], leading to

$$
\omega_{2 m}=C_{2 m-1}^{(\alpha, k)}, \quad \omega_{2 m+1}=C_{2 m}^{(\alpha, k)} .
$$

The following theorem is seen to be of the same form as Theorem 3.1 . 
Theorem 4.1. Let $A_{n}$ satisfy the conditions of (1.1). Then as $n \rightarrow \infty$ for fixed $m \geq 1$, we have

$$
\begin{aligned}
\rho_{2 m, n}^{(\alpha, k)} & \sim A+n^{-\alpha-2 m} \sum_{j=0}^{\infty} b_{m, j} n^{-j}, \quad k=1 \\
& \sim A+n^{-\alpha-m / k} \sum_{m=0}^{\infty} c_{m, j} n^{-j / k}, \quad k \geq 2,
\end{aligned}
$$

and

$$
\begin{aligned}
\rho_{2 m-1, n}^{\alpha, k)} & \sim n^{\alpha+2 m-1} \sum_{j=0}^{\infty} d_{m, j} n^{-j}, \quad k=1 \\
& \sim n^{\alpha+(m-1) / k+1} \sum_{j=0}^{\infty} e_{m, j} n^{-j}, \quad k \geq 2,
\end{aligned}
$$

where $b_{m, 0}, c_{m, 0}, d_{m, 0}$, and $e_{m, 0}$ may vanish for some values of $m$.

Proof. From (4.2) and (2.5), we find that

$$
\rho_{1, n}^{(\alpha, k)}=\alpha / \Delta A_{n}
$$

and

$$
\rho_{2, n}^{(\alpha, k)}=A_{n+1}+\frac{\alpha+1}{\alpha}\left[\Delta\left(1 / \Delta A_{n}\right)\right]^{-1}=e_{1}^{(\alpha+1)}\left(A_{n+1}\right) .
$$

It follows from Theorem 2.1 and from (2.8), (4.8), and (4.9) that (4.6) and (4.7) hold for $m=1$.

Let (4.6) and (4.7) hold for a given $m \geq 1$ with $b_{m, 0} \neq 0$ and $c_{m, 0} \neq 0$. Then it follows from (4.2) and (4.6) that (4.7) holds for $m+1$ with $d_{m+1,0}=$ $1 / b_{m, 0}$ and $e_{m+1}=1 / c_{m, 0}$. In order to show that (4.6) holds for $m+1$, we have

$$
\rho_{2 m+2, n}^{(\alpha, k)}=\rho_{2 m, n}^{(\alpha, k)}+C_{2 m+2}^{(\alpha, k)}\left[\Delta \rho_{2 m-1, n}^{(\alpha, k)}+C_{2 m+1}^{(\alpha, k)} \Delta\left(1 / \Delta \rho_{2 m, n}^{(\alpha, k)}\right)\right]^{-1}
$$

from (4.2). From (4.6) and (4.7), we find that

$$
\begin{aligned}
& {\left[\Delta \rho_{2 m-1, n}^{(\alpha, k)}+C_{2 m+1}^{(\alpha, k)} \Delta\left(1 / \Delta \rho_{2 m, n}^{(\alpha, k)}\right)\right]^{-1}} \\
& \quad=\left[C_{2 m+1}^{(\alpha, k)} \Delta\left(1 / \Delta \rho_{2 m, n}^{(\alpha, k)}\right)\right]^{-1}\left\{1+\Delta \rho_{2 m-1, n}^{(\alpha, k)}\left[C_{2 m+1}^{(\alpha, k)} \Delta\left(1 / \rho_{2 m, n}^{(\alpha, k)}\right)\right]^{-1}\right\}^{-1} \\
& \quad \sim\left[C_{2 m+1}^{(\alpha, k)} \Delta\left(1 / \Delta \rho_{2 m, n}^{(\alpha, k)}\right)\right]^{-1}\left[1+n^{-2} \sum_{j=0}^{\infty} \bar{c}_{m, j} n^{-j}\right], \quad k=1 \\
& \quad \sim\left[C_{2 m+1}^{(\alpha, k)} \Delta\left(1 / \Delta \rho_{2 m, n}^{(\alpha, k)}\right)\right]^{-1}\left[1+n^{-1 / k} \sum_{j=0}^{\infty} \bar{d}_{m, j} n^{-j / k}\right], \quad k \geq 2,
\end{aligned}
$$

where

$$
\begin{aligned}
\bar{c}_{m, 0} & =\frac{\alpha+2 m-1}{\alpha+2 m+1} b_{m, 0} d_{m, 0}, \quad k=1, \\
\bar{d}_{m, 0} & =\frac{\alpha+(m-1) / k+1}{\alpha+m / k+1} c_{m, 0} e_{m, 0}, \quad k \geq 2
\end{aligned}
$$


From (4.10), (4.11), and (2.6), we have

$$
\begin{aligned}
\rho_{2 m+2, n}^{(\alpha, k)} & \sim e_{1}^{(\alpha+2 m+1)}\left(\rho_{2 m, n+1}^{(\alpha, k)}\right)+n^{-\alpha-2 m-2} \sum_{j=0}^{\infty} \bar{e}_{m, j} n^{-j}, \quad k=1 \\
& \sim e_{1}^{(\alpha+m / k+1)}\left(\rho_{2 m, n+1}^{(\alpha, k)}\right)+n^{-\alpha-(m+1) / k} \sum_{j=0}^{\infty} \bar{f}_{m, j} n^{-j / k}, \quad k \geq 2,
\end{aligned}
$$

where

$$
\begin{aligned}
\bar{e}_{m, 0} & =-\frac{\alpha+2 m-1}{\alpha+2 m} b_{m, 0}^{2} d_{m, 0}, \\
\bar{f}_{m, 0} & =-\frac{\alpha+(m-1) / k+1}{\alpha+m / k+1} c_{m, 0}^{2} e_{m, 0} .
\end{aligned}
$$

Finally, it follows from (4.13) and Theorem 2.1 that (4.6) holds for $m+1$. Since (4.6) and (4.7) hold for $m=1$, they also hold up to the first value of $m$ for which $b_{m, 0}$ and $c_{m, 0}$ vanish. The remainder of the proof is now the same as that of Theorem 3.1 when $\theta_{m}^{n}$ is replaced by $\rho_{m, n}^{(\alpha, k)}$.

\section{THE LEVIN TRANSFORMATIONS}

Transformations of the Levin type [9] can be written in the form

$$
T_{m, n}=\frac{\Delta^{m}\left(n^{m-1} A_{n} / R_{n}\right)}{\Delta^{m}\left(n^{m-1} / R_{n}\right)}
$$

[14], where $R_{0}=1$ and $R_{n}=\Delta A_{n-1}, n \geq 1$, in the case of the $u$-transform, and $R_{n}=-\left[\Delta\left(1 / \Delta A_{n-1}\right)\right]^{-1}, n \geq 1$, in the case of the $v$-transform. When $A_{n}$ satisfies the conditions of (1.1), we find that these choices of $R_{n}$ are both asymptotically of the form

$$
R_{n} \sim n^{-\alpha} \sum_{j=0}^{\infty} r_{j} n^{-j / k}
$$

as $n \rightarrow \infty$, with $r_{0}=-\alpha a_{0}$ and $r_{0}=-\alpha a_{0} /(\alpha+1)$, respectively. The following theorem will be proved for general $R_{n}$ satisfying (5.2), with $r_{0} \neq 0$. Hence, the $u$ - and $v$-transforms are included as special cases. We see that (5.1) is a nonlinear transformation when the $r_{j}$ are functions of the coefficients $a_{j}$, as in the cases of the $u$ - and $v$-transforms, and that it is a linear transformation when the $r_{j}$ are given. When $k=1$, the statement of Theorem 5.1 is the same as that of Theorem 4.2 of Sidi in [14]. When $k=2$, however, we find that all sequences $T_{m, n}$ have the same rate of convergence for $m \geq 2$ as $n \rightarrow \infty$. We note that the differentiability conditions on the function $f(z)$ in [14] are needed only for Process II.

Theorem 5.1. Let $A_{n}$ satisfy the conditions of (1.1), and let $R_{n}$ satisfy (5.2) with $r_{0} \neq 0$. Then as $n \rightarrow \infty$ for fixed $m \geq 1$, we have

$$
\begin{aligned}
T_{m, n} & \sim A+n^{-\alpha-m} \sum_{j=0}^{\infty} b_{m, j} n^{-j}, \quad k=1 \\
& \sim A+n^{-\alpha-1 / k} \sum_{j=0}^{\infty} c_{m, j} n^{-j / k}, \quad k \geq 2,
\end{aligned}
$$


where $b_{m, 0}$ and $c_{m, 0}$ may vanish for some values of $m$.

Proof. From (1.1) and (5.2), we have

$$
n^{m-1} A_{n} / R_{n} \sim n^{m-1} A / R_{n}+n^{m-1} \sum_{j=0}^{\infty} d_{j} n^{-j / k}
$$

and

$$
n^{m-1} / R_{n} \sim n^{\alpha+m-1} \sum_{j=0}^{\infty} e_{j} n^{-j / k}
$$

for $k \geq 1$, where $d_{0}=a_{0} / r_{0}$ and $e_{0}=1 / r_{0}$. It follows that

$$
T_{m, n} \sim A+\frac{\Delta^{m}\left[n^{m-1} \sum_{j=0}^{\infty} d_{j} n^{-j / k}\right]}{\Delta^{m}\left[n^{\alpha+m-1} \sum_{j=0}^{\infty} e_{j} n^{-j / k}\right]} .
$$

We find that

$$
\begin{aligned}
\Delta^{m}\left[n^{m-1} \sum_{j=0}^{\infty} d_{j} n^{-j / k}\right] & \sim n^{-m-1} \sum_{j=0}^{\infty} f_{m, j} n^{-j}, \quad k=1 \\
& \sim n^{-1-/ k} \sum_{j=0}^{\infty} g_{m, j} n^{-j / k}, \quad k \geq 2,
\end{aligned}
$$

and that

$$
\Delta^{m}\left[n^{\alpha+m-1} \sum_{j=0}^{\infty} e_{j} n^{-j / k}\right] \sim n^{\alpha-1} \sum_{j=0}^{\infty} h_{m, j} n^{-j / k}
$$

for $k \geq 1$, where

$$
\begin{aligned}
& f_{m, 0}=(-1)^{m} m ! d_{m}, \\
& g_{m, 0}=-(m-1 / k-1)(m-1 / k-2) \cdots(1 / k) d_{1}, \\
& h_{m, 0}=(\alpha+m-1)(\alpha+m-2) \cdots \alpha e_{0} .
\end{aligned}
$$

Finally, (5.3) follows from (5.6) through (5.8) with

$$
b_{m, 0}=f_{m, 0} / h_{m, 0}, \quad c_{m, 0}=g_{m, 0} / h_{m, 0} .
$$

We see that $b_{m, 0}=0$ when $d_{m}$ vanishes, and that $c_{m, 0}=0$ when $d_{1}$ vanishes.

\section{A generalization of the Neville table}

The Neville table for a sequence $A_{n}$ is defined by

$$
e_{n}^{0}=A_{n}, \quad n \geq 0,
$$

and

$$
e_{n}^{m}=\left[n e_{n}^{m-1}-(n-m) e_{n-1}^{m-1}\right] / m,
$$

$m \geq 1, n \geq m$ [7, p. 191]. As in [7], the Neville table is found to accelerate the convergence of sequences which are asymptotically of the form (1.1) with $\alpha=1$ 
and $k=1$ as $n \rightarrow \infty$ for fixed $m$. Noting that $(n-\beta)(n-1)^{-\beta}=n^{1-\beta}+$ $O\left(n^{-1-\beta}\right)$ for $\beta \neq 0$, we arrive at the more general sequence transformation

$$
e_{0, n}^{(\alpha, k)}=A_{n}, \quad n \geq 0,
$$

and

$$
e_{m, n}^{(\alpha, k)}=\left[n e_{m-1, n}^{(\alpha, k)}-(n-\alpha-(m-1) / k) e_{m-1, n-1}^{(\alpha, k)}\right] /(\alpha+(m-1) / k),
$$

$m \geq 1, n \geq m$, and $k \geq 1$. We see that (6.4) reduces to (6.2) for $\alpha=1$, $k=1$. We will now show that this transformation accelerates the convergence of the family (1.1) when $k \geq 1$.

Theorem 6.1. Let $A_{n}$ satisfy the conditions of (1.1). Then as $n \rightarrow \infty$ for fixed $m \geq 0$, we have

$$
e_{m, n}^{(\alpha, k)} \sim A+n^{-\alpha-m / k} \sum_{j=0}^{\infty} b_{m, j} n^{-j / k}
$$

for $k \geq 1$, where $b_{m, 0}$ may vanish for some values of $m$.

Proof. Assuming that (6.5) holds for a given $m \geq 0$, we find from (6.4) that

$$
e_{m+1, n}^{(\alpha, k)} \sim A+\left\{b_{m, 0}\left[n^{-\alpha-m / k+1}-(n-\alpha-m / k)(n-1)^{-\alpha-m / k}\right]\right.
$$

$$
\begin{aligned}
& +\sum_{j=1}^{\infty} b_{m, j}\left[n^{-\alpha-m / k-j / k+1}-(n-1)^{-\alpha-m / k-j / k+1}\right] \\
& \left.+(\alpha+m / k-1) \sum_{j=1}^{\infty} b_{m, j}(n-1)^{-\alpha-m / k-j / k}\right\} /(\alpha+m / k) .
\end{aligned}
$$

We have

$$
\begin{aligned}
n^{-\alpha-m / k+1} & -(n-\alpha-m / k)(n-1)^{-\alpha-m / k} \\
& \sim n^{-\alpha-m / k-1} \sum_{j=0}^{\infty} c_{m, j} n^{-j} \\
& \sim n^{-\alpha-(m+1) / k} \sum_{j=0}^{\infty} c_{m, j} n^{-[(j+1) k-1] / k},
\end{aligned}
$$

where

$$
c_{m, 0}=(\alpha+m / k)(\alpha+m / k-1) / 2,
$$

and we can verify that the second and third terms on the right-hand side of (6.6) are asymptotically of the form of the second term on the right of (6.5) with $m$ replaced by $m+1$. It follows that $e_{m+1, n}^{(\alpha, k)}$ is asymptotically of the form of (6.5) with

$$
\begin{aligned}
b_{m+1,0} & =\left(b_{m, 0} c_{m, 0}-b_{m, 1}\right) /(\alpha+m), \quad k=1 \\
& =-b_{m, 1} /(\alpha k+m), \quad k \geq 2 .
\end{aligned}
$$

Hence, since $\alpha+m / k \neq 0, b_{m+1,0}$ may be either nonzero or zero, and the statement of Theorem 6.1 holds. 


\section{NUMERICAL EXAMPLES}

In order to test the preceding asymptotic results, the six sequence transformations considered have been applied to the sequence

$$
A_{n}=A-\frac{n^{-\alpha}}{1+n^{-1 / k}}
$$

for $k=1,2$, and 3, and for both real and complex values of $\alpha$ and $A$. Also, calculations have been carried out by the algorithm and FORTRAN program of [6] with $\sigma=1,1.1$, and 1.2 , and $m=1,2$, and 3. Calculations were carried out on the CDC Cyber 875 in double precision, or 28 significant figures, when $A_{n}$ is real. When $A_{n}$ is complex, calculations were carried out on a personal computer in double precision, or about 16 significant figures, using RM/FORTRAN. The number of terms used in each calculation was 49 , but the later terms were not always useful because of loss of accuracy. This loss of accuracy may not be present in the case of the $d^{(m)}$-transformation with $\sigma>1$, however, since the latter is then found to become increasingly more stable numerically as $\sigma>1$ increases, and to give more accurate results than for $\sigma=1$.

Table 7.1 gives the number of significant figures obtained by each of the six transformations when applied to (7.1) for $\alpha=1 / 2$ and $k=1,2$, and 3, and for the algorithm of [6]. In the iteration of $e_{1}^{(s)},(2.18)$ was used when $k=1$, and (2.17) when $k=2$ and 3. In the algorithm of [6], $\sigma=1$ and $m=k$ for $k=1,2$, and 3. More accurate results can be obtained for $\sigma=1.1$ and 1.2 than for $\sigma=1$, but more terms of $A_{n}$ are then used in the calculation.

The numerical behavior of the first six of the transformed sequences in Table 7.1 was found to be consistent with Theorems 2.1 through 6.1 when $m$ is not too large. For larger values of $m$, the maximum accuracy for fixed $m$ does not necessarily occur at the largest value of $n$ due to loss of accuracy. Also, the maximum accuracy does not necessarily occur at the largest value of $m$. We see that the algorithm of [6] gives good accuracy for each of the values of $k$.

Tables 7.2 through 7.5 illustrate the asymptotic behavior of $W^{m}\left(A_{n}\right)$ and of the $u$-transform as given in Theorems 2.2 and 5.1, respectively. As in $\S 5$,

TABLE 7.1. Number of significant digits obtained in acceleration of convergence of (7.1) with $\alpha=1 / 2$ and $A=1$

\begin{tabular}{cccc}
\hline Method & $k=1$ & $k=2$ & $k=3$ \\
\hline Iter. of $e_{1}^{(s)}$ & 18 & 11 & 9 \\
$W^{m}\left(A_{n}\right)$ & 15 & 12 & 7 \\
$\theta_{m}^{(n)}$ & 14 & 9 & 5 \\
$\rho_{m, n}^{(\alpha, k)}$ & 22 & 11 & 9 \\
$u$-transform & 16 & 3 & 3 \\
$e_{m, n}^{(\alpha, k)}$ & 19 & 12 & 8 \\
$d^{(k)}$-transformation & 16 & 13 & 9 \\
\hline
\end{tabular}


TABLE 7.2. Calculated values of $B_{m, n}=\left[W^{m}\left(A_{n}\right)-1\right] n^{1 / 2+2 m}$ for (7.1) with $k=1$

\begin{tabular}{cccc}
\hline$n$ & $B_{1, n-3}$ & $B_{2, n-6}$ & $B_{3, n-9}$ \\
\hline 47 & -4.92723 & -4.22842 & -3.66563 \\
48 & -4.93275 & -4.23681 & -3.77912 \\
49 & -4.93803 & -4.24470 & -3.89092 \\
\hline
\end{tabular}

TABLE 7.3. Calculated values of $C_{m, n}=\left[W^{m}\left(A_{n}\right)-1\right] n^{1 / 2+m / k}$ for (7.1) with $k=2$

\begin{tabular}{cccc}
\hline$n$ & $C_{1, n-3}$ & $C_{2, n-6}$ & $C_{3, n-9}$ \\
\hline 47 & 0.318003 & -0.0035669 & -0.0000664 \\
48 & 0.318328 & -0.0034819 & -0.0000651 \\
49 & 0.318638 & -0.0034005 & -0.0000639 \\
\hline
\end{tabular}

TABLE 7.4. Calculated values of $D_{m, n}=\left(T_{m, n}-1\right) n^{1 / 2+m}$ for (7.1) with $k=1$

\begin{tabular}{llll}
\hline$n$ & $D_{1, n-1}$ & $D_{2, n-2}$ & $D_{3, n-3}$ \\
\hline 47 & 2.69032 & -5.21871 & 9.48604 \\
48 & 2.68576 & -5.21782 & 9.49511 \\
49 & 2.68142 & -5.21695 & 9.50380 \\
\hline
\end{tabular}

TABLE 7.5. Calculated values of $E_{m, n}=\left(T_{m, n}-1\right) n^{1 / 2+1 / k}$ for (7.1) with $k=2$

\begin{tabular}{cccc}
\hline$n$ & $E_{1, n-1}$ & $E_{2, n-2}$ & $E_{3, n-3}$ \\
\hline 47 & 0.737337 & 0.325399 & 0.190261 \\
48 & 0.740541 & 0.325562 & 0.190261 \\
49 & 0.743638 & 0.325719 & 0.190674 \\
\hline
\end{tabular}

the $u$-transform is denoted by $T_{m, n}$. We see that the calculated quantities in a given column become nearly constant as $n$ increases, in agreement with these theorems.

In order to investigate the acceleration of convergence of the algorithm of [6] numerically when applied to (7.1), we repeat the computations of Table 7.5 for the latter when $m=k=2$ and $\sigma=1$. Denoting this transformation by $U_{m, n}$, we obtain the results shown in Table 7.6. This table indicates that acceleration of convergence under Process $I$ is not significant, and hence, that the acceleration of convergence found in Table 7.1 for $k=2$ appears to occur mainly under Process II. The corresponding calculations for $m=k=3$ and $\sigma=1$ lead to these same conclusions when $k=3$. 
TABLE 7.6. Calculated values of $F_{m, n}=\left(U_{m, n}-1\right) n^{1 / 2+1 / k}$ for (7.1) with $k=2$

\begin{tabular}{cccc}
\hline$n$ & $F_{1, n-1}$ & $F_{2, n-2}$ & $F_{3, n-3}$ \\
\hline 47 & 0.737337 & 0.115339 & 0.282021 \\
48 & 0.740541 & 0.114142 & 0.280211 \\
49 & 0.743638 & 0.112984 & 0.278329 \\
\hline
\end{tabular}

TABLE 7.7. Number of significant digits obtained in acceleration of convergence of (7.1) with $\alpha=1 / 2+i$ and $A=1+i$

\begin{tabular}{cccc}
\hline Method & $k=1$ & $k=2$ & $k=3$ \\
\hline Iter. of $e_{1}^{(s)}$ & 12 & 7 & 6 \\
$W^{m}\left(A_{n}\right)$ & 9 & 5 & 5 \\
$\theta_{m}^{(n)}$ & 9 & 5 & 5 \\
$\rho_{m, n}^{(\alpha, k)}$ & 13 & 7 & 6 \\
$u$-transform & 10 & 4 & 5 \\
$e_{m, n}^{(\alpha, k)}$ & 11 & 7 & 6 \\
$d^{(k)}$-transformation & 11 & 7 & 6 \\
\hline
\end{tabular}

TABLE 7.8. Number of significant digits obtained in the acceleration of convergence of (7.1) with $A=1+i, k=1$

\begin{tabular}{cccc}
\hline Method & $\alpha=1 / 2+10 i$ & $\alpha=1 / 2+25 i$ & $\alpha=1 / 2+50 i$ \\
\hline Iter. of $e_{1}^{(s)}$ & 16 & 16 & 16 \\
$W^{m}\left(A_{n}\right)$ & 12 & 15 & 16 \\
$\theta_{m}^{(n)}$ & 13 & 15 & 16 \\
$\rho_{m, n}^{(\alpha, k)}$ & 14 & 15 & 16 \\
$u$-transform & 13 & 15 & 16 \\
$e_{m, n}^{(\alpha, k)}$ & 9 & 9 & 4 \\
$d^{(1)}$-transformation & 13 & 14 & 16 \\
\hline
\end{tabular}

Table 7.7 gives the number of significant figures obtained when the six transformations considered and the algorithm of [6] are applied to (7.1) with the complex parameters $\alpha=1 / 2+i$ and $A=1+i$ and with $k=1,2$, and 3 . The smaller of the number of significant digits in the real and imaginary parts is given. As before, the $d^{(k)}$-transformation gives good accuracy for each of the values of $k$. Similarly, Table 7.8 gives the accuracy obtained with these same transformations when $A=1+i, k=1$, and $\alpha=1 / 2+10 i, 1 / 2+25 i$, and $1 / 2+50 i$. The difference between the $u$-transform and the $d^{(1)}$-transformation is due only to differences in loss of accuracy.

Table 7.8 shows that the generalized Neville table is the most sensitive of the six transformations to the magnitude of $\alpha$ in this example, and suggests that it should be used only for small or moderate values of $|\alpha|$. 
As additional examples, we apply the generalized $\rho$-algorithm to the sequences

$$
A_{n}=\sum_{j=1}^{n}\left(j+e^{1 / j}\right)^{-\sqrt{2}}
$$

and

$$
A_{n}=\sum_{j=1}^{n}\left(\begin{array}{c}
-\frac{1}{2} \\
j-1
\end{array}\right) \frac{(-1)^{j+1}}{4 j-3}
$$

of [16], where they were found not to be accelerated by the $\rho$-algorithm. By use of the Euler-Maclaurin summation formula, it can be shown that these sequences satisfy the conditions of (1.1) with $k=1$ and with $\alpha=\sqrt{2}-1$ in (7.2) and $\alpha=1 / 2$ in (7.3). It follows from Theorem 4.1 that the convergence of these sequences is accelerated by the generalized $\rho$-algorithm with $k=1$ and with $\alpha=\sqrt{2}-1$ and $1 / 2$, respectively, and that their convergence is not accelerated by the original $\rho$-algorithm under the limiting process $n \rightarrow \infty$ for constant $m$. Calculations for (7.2) and (7.3) have been carried out in double precision on the CDC 875 , using 49 terms of each sequence. The results obtained converged to 19 and 20 significant figures, respectively, in the case of the generalized $\rho$-algorithm, and to 2 and 3 significant figures, respectively, in the case of the original $\rho$-algorithm.

Finally, we note that Example 2 of [12] satisfies (1.1) with $\alpha=1 / 2$ and $k=2$. Applying the generalized $\rho$-algorithm to 49 terms of the sequence, we obtain 11 significant figures when $\alpha=1 / 2$ and $k=2$, and 5 significant figures when $\alpha=1 / 2$ and $k=1$.

\section{BIBLIOGRAPHY}

1. P. Bjørstad, G. Dahlquist, and E. Grosse, Extrapolation of asymptotic expansions by a modified Aitken $\delta^{2}$-formula, BIT 8 (1981), 56-65.

2. C. Brezinski, Études sur les $\varepsilon$ - et $\rho$-algorithmes, Numer. Math. 17 (1971), 153-162.

3. _ـ Accélération de suites à convergence logarithmique, C. R. Acad. Sci. Paris Ser A-B 273 (1971), A727-A730.

4. _ Some new convergence acceleration methods, Math. Comp. 39 (1982), 133-145.

5. T. Fessler, W. F. Ford, and D. A. Smith, HURRY: An acceleration algorithm for scalar sequences and series, ACM Trans. Math. Software 9 (1983), 346-354.

6. W. F. Ford and A. Sidi, An algorithm for a generalization of the Richardson extrapolation process, SIAM J. Numer. Anal. 24 (1987), 1212-1232.

7. D. S. Gaunt and A. J. Guttmann, Asymptotic analysis of coefficients, phase transitions and critical phenomena, Vol. 3, Series Expansions for Lattice Models (C. Domb and M. S. Green, eds.), Academic Press, New York, 1974, pp. 181-243.

8. C. Hunter and B. Guerrieri, Deducing the properties of singularities of functions from their Taylor series coefficients, SIAM J. Appl. Math. 39 (1980), 248-263.

9. D. Levin, Development of non-linear transformations for improving convergence of sequences, Internat. J. Comput. Math. B3 (1973), 371-388.

10. D. Levin and A. Sidi, Two new classes of nonlinear transformations for accelerating the convergence of infinite integrals and series, Appl. Math. Comput. 9 (1981), 175-215.

11. S. Lubkin, A method of summing infinite series, J. Res. Nat. Bur. Standards 48 (1952), 228-254. 
12. N. Osada, A convergence acceleration method for some logarithmically convergent sequences, SIAM J. Numer. Anal. 27 (1990), 178-189.

13. D. Shanks, Non-linear transformations of divergent and slowly convergent sequences, J. Math. and Phys. 34 (1955), 1-42.

14. A. Sidi, Convergence properties of some nonlinear sequence transformations, Math. Comp. 33 (1979), 315-326.

15. Analysis of convergence of the T-transformation for power series, Math. Comp. 35 (1980), 833-850.

16. D. A. Smith and W. F. Ford, Acceleration of linear and logarithmic convergence, SIAM J. Numer. Anal. 16 (1979), 223-240.

17. D. A. Smith and W. F. Ford, Numerical comparisons of nonlinear convergence accelerators, Math. Comp. 38 (1982), 481-499.

18. A. Van Tuyl, Application of methods for acceleration of convergence to the calculation of singularities of transonic flows, Padé Approximants Method and its Applications to Mechanics, Lecture Notes in Phys., No. 47, Springer-Verlag, Berlin, 1976, 209-223.

19. J. Wimp, Some transformations of monotone sequences, Math. Comp. 26 (1972), 251-254.

20. mer. Math. 23 (1974), 1-17.

21. P. Wynn, On a device for computing the $e_{m}\left(S_{n}\right)$ transformation, MTAC 10 (1956), 91-96.

22. __ On a Procrustean technique for numerical transformation of slowly convergent sequences and series, Proc. Cambridge Philos. Soc. 52 (1956), 663-671.

Applied Mathematics Branch, Code R44, Naval Surface Warfare Center, Silver SPRING, MARYLAND 20903-5000

Current address: $1000 \mathrm{~W}$. Nolcrest Drive, Silver Spring, Maryland 20903

E-mail address: avt@clark.net 